\title{
Diffusion flames in condensed-phase energetic materials: Application to Titanium-Boron combustion
}

\author{
Sushilkumar P. Koundinyan, John B. Bdzil, Moshe Matalon, and D. Scott Stewart ${ }^{1}$ \\ Mechanical Science and Engineering, University of Illinois, Urbana, IL
}

\begin{abstract}
The characteristics of a steady diffusion flame that arises at the interfaces of two condensed phase reactant streams that form an opposed counterflow are discussed. We assume that the flow is due to deformation from compaction or local heating and thermal expansion processes in the microscale environment of composite energetic materials. As a representative example of high temperature combustion of metal/intermetallic reactants, the overall reaction of titanium and boron to create titanium diboride products is considered under near isobaric conditions. The multi-component diffusion description uses a generalized Fick formulation with coefficients related to the binary diffusivities defined in the Maxwell-Stefan relations. A fairly simple depletion form with Arrhenius temperature dependent coefficients is used to describe the reaction rate. Several types of analyses are carried out at increasing levels of complexities: an asymptotic analysis valid in the limit of low strain rates (high residence time in the reaction zone), a constant mixture density assumption that simplifies the flow description, diffusion models with equal and unequal molecular weights for the various species, and a full numerical study for finite rate chemistry, composition-dependent density and strain rates extending from low to moderate values. All are found to agree remarkably well in describing the flame structure, the flame temperature and the degree of incomplete combustion. Of particular importance is the determination of a critical strain rate beyond which steady burning may no longer be observed. The analysis has a general character and can be applied to other condensed phase energetic material systems, where reaction and diffusion occur in the presence of flow and material deformation.
\end{abstract}

\section{(c) 2015 Published by Elsevier Ltd.}

Keywords: Condensed phase combustion, Extinction, Diffusion flame, Titanium-Boron 2010 MSC: 00-01, 99-00

\section{Introduction}

Energetic materials are a broad class of manufactured materials that traditionally comprise both propellants and explosives, but include thermite and intermetallic/metal mixtures as well. They consist of molecular explosives and molecular oxidizer crystallites, like HMX and Ammonium perchlorate (AP), metal elements like aluminum ( $\mathrm{Al})$ and

\footnotetext{
${ }^{1}$ Corresponding author. Email: dss@illinois.edu
} 
titanium (Ti), metal oxides like iron oxide, copper oxide, intermetallic elements like carbon, silicon and boron, and plastic binders and resins like HTPB. The mixtures are made from elements, or a composite of components that have been processed and have imperfections and contaminants, such as cracks or inclusions, or have been subjected to surface oxidation, as in propellants. The components in the mixture prior to the composite assembly, all have their own individual mechanical and thermo-chemical identity.

Explosive compounds are pre-mixed at the molecular level, whereas the mixtures of metals, metal oxides and intermetallics compound are initially separated constituents that react in the vicinity of the initial material boundaries, enabled by diffusion of reactants and products through the molecular condensed phase regions. Typically, the individual components are combined to make an agglomerated composite mixture of powders and other ingredients. The powders have a particle size distribution, with dimension that varies from one to several hundreds microns, and characteristic particle morphology. The mixtures are pressed or cast into a mold for applications.

Reactive energetic materials, as defined in a 2004 US National Academy of Sciences report [1], generally combine two or more inert solids, and an ignition source is required to start the chemical reaction at the interfaces of the initially separated reactants. Agglomerated composites can be ignited by shock compression if the components comprising the initial reactive mixture are not highly dense. The conditions required for ignition occur through the collapse of interstitial voids, typically of initial volume fraction in the range of 1 to $20 \%$. During the void collapse, thermomechanical flows and material displacements occur due to the densification caused by the shock confinement, and lead to large strain rates in the reactive material components. The resulting deformations are associated with energy dissipation that cause localized heating (hot spots) which, in turn, generates thermal events that are needed for melting the constituent components and to initiate the reaction at the material interfaces. The localized thermal events in the mixture also lead to local streaming motions and greater species mobility of reactants.

Energetic materials fall generally into two broad classes based on how they are manufactured: (i) agglomerated composite mixtures of powders and other reactive components, and (ii) finely-spaced, structured or layered composites with specified interstitial spacing between the reactive components. The current state of experimental investigations and modeling of finely-spaced multilayered reactive materials has been recently reviewed by Rogachev [2], Weihs [3] and Adams [4]. A typical illustration is described by Weihs [5], where approximately 10 micron layers of foil (of 
say, nickel and aluminum) are pressed into a laminate ply. The formation of nickel/aluminum products, for example, starts with thermal initiation (heating) of the composite material that first melts the aluminum and allows the chemical reaction to proceed. The modeling effort focuses on the effect of the laminate bilayer thickness on ignition and on the velocity of the self-propagating reactions of a number of reactive material pairs. In general, steady self-propagation of a reacting front in the laminate material moves perpendicular to the normal of the planes of laminate plies. The speed of the the steady self-propagation reaction front is found to be dependent on the ply spacing and composition [6]. For example, Sraj et.al [7] studied the response of Ni/Al multilayered composites to shock compression; he used the Sandia CTH hydrocode to simulate the mechanical deformation of layered Ni/Al material to shock compression and investigate the effects of the bilayer thickness and the shock velocity and orientation on the combustion process. When the normal to the plane of the impact that creates the shock is in the same direction as the normal to the plane of the laminate plies, the propagation process is found to be highly unsteady.

An alternative to the well-structured layered composites are agglomerated materials made of a mixture of reactive and inert components. Glumac and Stewart [8] have recently reported results of shock compaction experiments on porous materials that are initially composed of two reactive components. Systems that have been studied include the aluminum ( $\mathrm{Al})$, copper oxide $(\mathrm{CuO})$ thermite, and the metal/intermetallic system composed of titanium, silicon, and titanium, boron. A typical shock compaction experiment is carried out for $80 \%$ porous, stoichiometric mixture of components, with the initial mass fractions based on the overall equilibrium products. For the $\mathrm{Al}, \mathrm{CuO}$ system the stoichiometric reaction is $2 \mathrm{Al}+3 \mathrm{CuO} \rightarrow \mathrm{Al}_{2} \mathrm{O}_{3}+3 \mathrm{Cu}$. The reactive material sample is placed in a striker assembly, and compacted by the action of two metal bars that are shock loaded by the firing of detonators on each end. A sustained heterogeneous front was found to propagate at an average speed of approximately $6-20 \mathrm{~cm} / \mathrm{sec}$. High speed microscopic photography was used to record the emitted light seen through a small observation window. On a length scale of 10 to $20 \mu \mathrm{m}$ one observes the sudden formation and disappearance of intense spots of light, corresponding to intense and weak chemical reactions recurring within a time interval of approximately $100 \mu$ s. Similar results were observed for the titanium - silicon system and more extensive experiments are planned for the titanium - boron system. These experiments clearly demonstrate that the overall combustion process is highly unsteady. While the lead reactive front after shock compaction is observed to propagate at a well-defined average velocity, measurable and robust, time- 
dependent heterogeneous reaction-diffusion processes occur on the micro-scale, corresponding to the initial size of the reactive component particles, before and after the passage of the lead shock. Since the component materials and their reactants are very hot and experience significant thermal expansion, the chemically reacting material experiences a distribution of local flow velocities and strain rates, primarily at the material interface of the reacting components.

The theoretical modeling approach for the finely-space laminate, or regular structured materials, can be summarily described as an approach that lumps, or relies on cross-sectional averages for all transport phenomena, such as bulk heat transfer and diffusion, and for all material properties of the laminate/arrays [4]. These reduced models seem to appropriately describe observed phenomena that depend on average properties of the system, such as bulk temperature and reaction extent, or the self-propagation speed of a reactive front propagating along the axis of the plane of the plies. They do not explicitly describe the reaction-diffusion phenomena at material interfaces, and cannot delineate separate molecularly distinct reactants. The focus in this work is exactly on the processes taking place on a small-scale of the the initially-separated component materials that comprise the mixtures, that are not necessarily layered or structured. Our approach delineates separate molecularly distinct reactants and employs a multicomponent, thermodynamic formulation with separated reactants and products, each with their own properties. While we employ some simplifications, we do not use a lumped, averaged formulation in the same sense of the reduced models found in the analysis of finely-spaced laminates or arrays. Fundamental understanding of these local events will serve as a basis for future modeling of time-dependent reaction processes in both classes of energetic materials, agglomerated composites and finely-spaced, structured or layered composites.

In this paper we examine the reaction-diffusion processes in the mixing region of two opposed streams of distinct reactants, that stagnate on the stream axis as shown in Fig. 1. The flow due to material deformation, local heating, and thermal expansion processes, has been presumably established at the micro-scale by prior shock compaction events that we do not model; such considerations were given, for example, in the studies reported in [7, 9]. The flow of opposed streams is characterized by a single strain rate - the ratio of the average displacement speed to the length scale that characterizes the material nonuniformity - or, equivalently, the inverse of a residence time that we presume given. We show that in this counterflow configuration, a balance between advection, diffusion and chemical reaction is possible under steady conditions when the strain rate is not too large. Otherwise, the only possible balance is that 
of a nearly-frozen or weak burning state (not discussed in this paper). In the larger picture, we envision a cooperative time-dependent mechanism between local reaction sites that experience intense reaction and slow reaction at local interfaces, that depends on the local strain rate distribution in the composite material.

Although our analysis holds for any three-components system, we have chosen the titanium (Ti) and boron (B) system as a representative example of metal/intermetallic reactive material system. Their overall reaction, to create titanium diboride $\left(\mathrm{TiB}_{2}\right)$ products,

$$
\mathrm{Ti}+2 \mathrm{~B} \rightarrow \mathrm{TiB}_{2}
$$

is considered under nearly-isobaric conditions, such that the pressure is represented by the hydrostatic pressure. The equation of state of the three components and the formulation that defines the equilibrium equation of state of the mixture is based on multicomponent thermodynamics formulations that are similar to those used in the study of metallurgy and materials [10]. As such, the formulation is based on Gibbs thermodynamic potentials where one assumes that at each point of the condensed phase mixture, there is a single stress state and temperature. Each isolated component is assumed to have its own distinct reference density, and we neglect thermal expansion in the components. This is consistent with the notion that the change in composition due to reaction is much larger than changes due to thermal expansion. As a result the mechanical equation of state for the mixture takes a simple form whereby the specific volume of the mixture is simply a sum of the intrinsic densities weighted with the mass fraction of each component. This form of the mechanical equation of state stands in contrast with that for a mixture of reacting gases that is a relation between the specific volume, pressure, temperature and mass fractions. The diffusion model for the components is derived from an effective Fick diffusion formulation as described by Curtiss [11] and Curtiss and Bird [12], whereby a Maxwell-Stefan law formulated in terms of binary diffusivities is expressed as a generalized Fick diffusion law with symmetric diffusion coefficients. The resulting diffusion coefficients used in our model are then chosen to lead consistent results with experimental data reported by Trunov et al. [13].

The governing equations describing the steady burning of initially separated titanium and boron in a counterflow geometry are presented in the next section. These equations are addressed first under the assumption of constant mixture density, which enables the construction of an analytical solution in the fast chemistry limit. The expressions for flame position and temperature are used when compared to experimental data to estimate binary diffusion coefficients 
that are not well-known, and or measured under restricted conditions. We then address numerically the general case, of finite rate chemistry and when the density of the mixture varies reflecting the local composition of the mixtures. In particular, we derive extinction conditions that differentiate between vigorous and weak burning between titanium and boron. We note that the analytical solution, valid for large Damköhler numbers, is used to validate the numerical solution and calibrate the corresponding strain rates in deriving the extinction conditions.

\section{Formulation}

The counterflow geometry under consideration is shown in Fig. 1, where far to the left (state 1) there is only titanium and far to the right (state 2) there is only boron; the intrinsic densities are denoted by $\hat{\rho}_{1_{0}}$ and $\hat{\rho}_{2_{0}}$, respectively. Under steady conditions, the material deformation may be described by a velocity field $\mathbf{v}$ of the form $\mathbf{v}=(u(x), y \bar{v}(x))$. This "similarity solution" implies that the pressure gradient in the transverse direction $y$ is necessarily linear and admits planar combustion fronts such that all state variables, the mass fractions $Y_{i}$, the density $\rho$, and temperature $T$,

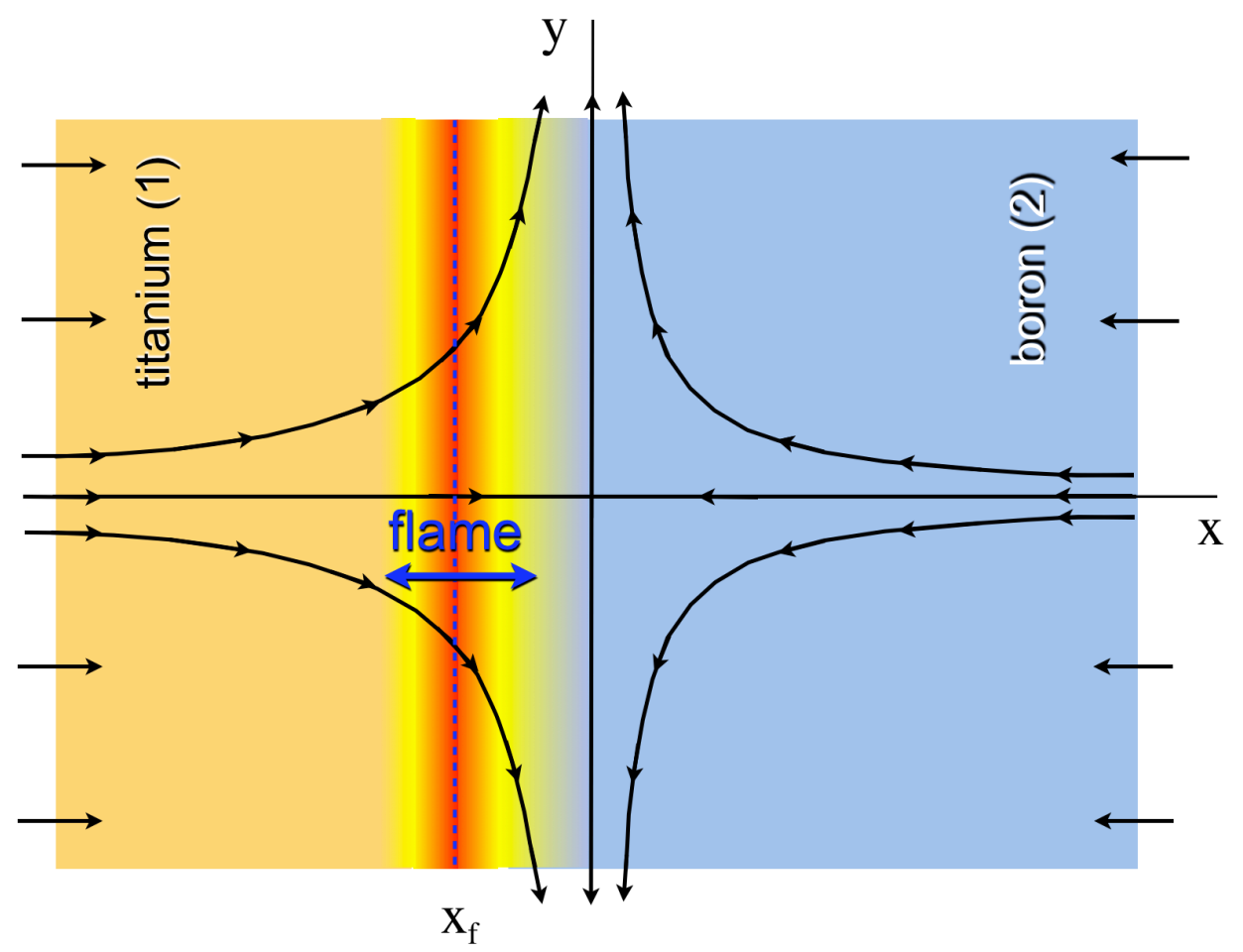

Figure 1. Schematic of the condensed phase counterflow diffusion flame, with titanium entering from the left and boron from the right. The color shades clearly delineate the planar mixing region and reaction zone. 
are functions of $x$ alone. The constituents in the combustion zone include titanium of mass fraction $Y_{1}$, boron of mass fraction $Y_{2}$, and titanium diboride products of mass fraction $Y_{3}$. Overall mass conservation implies that

$$
Y_{1}+Y_{2}+Y_{3}=1
$$

The conductivity $k$ and specific heat (at constant pressure) $c_{p}$ of the mixture (defined as mass-weighted averages) are, in general, functions of temperature but for simplicity they will be taken here as constants. Finally, the chemical reaction (1) between $\mathrm{Ti}$ and $\mathrm{B}$ is assumed to proceed at a rate

$$
\omega=\mathcal{B} Y_{1} Y_{2}^{2} e^{-E / R T}
$$

where $E$ is the activation energy, $\mathcal{R}$ is the universal gas constant and $\mathcal{B}$ is an appropriately defined pre-exponential factor. Different reaction orders could be considered without difficulty; the present form that simplifies the reaction to one-step process was made for simplicity.

\subsection{Conservation equations}

The governing equations, describing conservation of mass, momentum, and energy under steady conditions simplify to

$$
\begin{gathered}
\frac{d}{d x}(\rho u)+\rho \bar{v}=0 \\
\rho u \frac{d \bar{v}}{d x}+\rho \bar{v}^{2}=C \\
\rho u c_{p} \frac{d T}{d x}-k \frac{d^{2} T}{d x^{2}}=Q \omega \\
\rho u \frac{d Y_{1}}{d x}+\frac{d}{d x}\left(\rho Y_{1} V_{1}\right)=-W_{1} \omega \\
\rho u \frac{d Y_{2}}{d x}+\frac{d}{d x}\left(\rho Y_{2} V_{2}\right)=-2 W_{2} \omega
\end{gathered}
$$

where $V_{i}, W_{i}$ stand for the diffusion velocity and molecular weight of species $i$, and $Q$ is the overall heat release. As noted earlier, the pressure gradient in the transverse $y$-direction is linear, given by $\partial p / \partial y=-C y$, where $C$ is a constant determined by the far field boundary conditions. Specifically, equ. (5) implies that there is a relation between the densities and strain rates at the far ends, such that

$$
\hat{\rho}_{1_{0}} \epsilon_{1}^{2}=\hat{\rho}_{2_{0}} \epsilon_{2}^{2}=C .
$$


Hence the motion of the reactants impinging on each other is characterized by a single strain rate $\epsilon$ (in units of $1 / \mathrm{s}$ ) which we choose as $\epsilon=\epsilon_{1} / 2$; the factor of 2 is introduced solely to facilitate the form of the analytical asymptotic solution described below. The axial dependence of the pressure can be obtained a-posteriori by solving the $x$-component of the momentum equation (not written above).

Equations (4)-(8) must be supplemented with constitutive relations for the diffusion velocities and an equation of state for the mixture. Starting with the assumption that the Gibbs free energy of each component of the mixture can be summed, the Gibbs potential for the mixture which is a function of pressure, temperature, and mixture composition is

$$
g=\sum_{i=1}^{3} g_{i}(p, T) Y_{i},
$$

where the energies related to mixing have been neglected. The specific volume $v=1 / \rho$ of the mixture and individual components are given by the thermodynamic relation:

$$
v=\left.\frac{\partial g}{\partial p}\right|_{T, Y_{i}} \text { and } \quad v_{i}=\left.\frac{\partial g_{i}}{\partial p}\right|_{T}
$$

where $v_{i}$ are the partial volumes, or the volume of the components. This leads to

$$
v=\sum_{i=1}^{3} v_{i}(p, T) Y_{i}
$$

which is the mechanical equation of state for the mixture. (Note: The symbol $v$ represents the specific volume and should not be confused with $v$ which represents the y-component of velocity.) If we further assume that the volume change due to pressure variations is small, and neglect the effect of temperature, the partial volumes $v_{i}$ can be approximated by their reference values $\hat{v}_{i_{0}}$. The mechanical equation of state (12), when expressed in terms of the densities, then becomes

$$
\rho^{-1}=\sum_{i=1}^{3} Y_{i} \hat{\rho}_{i_{0}}^{-1}
$$

where $\hat{\rho}_{i_{0}}$ is the intrinsic density, and $\hat{\rho}_{i_{0}}^{-1}$ the intrinsic specific volume, of species $i$. Using (2) the equation of state simplifies to

$$
\frac{1}{\rho}=\frac{1}{\hat{\rho}_{3_{0}}}+\left(\frac{1}{\hat{\rho}_{1_{0}}}-\frac{1}{\hat{\rho}_{3_{0}}}\right) Y_{1}+\left(\frac{1}{\hat{\rho}_{2_{0}}}-\frac{1}{\hat{\rho}_{3_{0}}}\right) Y_{2}
$$

With the flow specified by the strain rate $\epsilon$ and the material properties far to the left (denoted by subscript 1) and 
far to the right (denoted by subscript 2) assumed uniform, the boundary conditions are:

$$
\begin{gathered}
d u / d x \sim-2 \epsilon \quad \text { as }|x| \rightarrow \infty, \\
\rho=\hat{\rho}_{1_{0}}, \quad Y_{1}=1, \quad Y_{2}=0, \quad T=T_{\infty} \quad \text { as } x \rightarrow-\infty, \\
\rho=\hat{\rho}_{2_{0}}, \quad Y_{1}=0, \quad Y_{2}=1, \quad T=T_{\infty} \quad \text { as } x \rightarrow+\infty .
\end{gathered}
$$

Note that there is no need to specify a condition for $\bar{v}$, because it is obtained from

$$
\bar{v}=-\frac{1}{\rho} \frac{d}{d x}(\rho u)
$$

by differentiation.

When density variations are small, the equation of state (14) can be effectively replaced by $\rho=$ constant, and the velocity field everywhere is given by

$$
u=-2 \epsilon x, \quad \bar{v}=2 \epsilon .
$$

The problem reduces to the reaction-diffusion system (6)-(8). The constant-density approximation will be used for simplicity in the asymptotic description described below. In general, variations in the density modify the overall velocity field which, in turn, affects the combustion field. In Section 4, numerical computations are carried out in order to assess the importance of density variations in condensed-phase combustion.

\subsection{Diffusion}

The most common expressions used for multi-component diffusion are the Maxwell-Stefan (MS) relations

$$
\nabla X_{i}=\sum_{j} \frac{X_{i} X_{j}}{\mathscr{D}_{i j}}\left(\mathbf{V}_{j}-\mathbf{V}_{i}\right),
$$

with the summation taken over all species present [14]; here $X_{i}$ is the molar fraction, $\mathbf{V}_{\mathbf{i}}$ is the diffusion velocity vector of species $i$, and $\mathscr{D}_{i j}=\mathscr{D}_{j i}$ is the binary diffusivity of a pair of species $(i, j)$. Although this relation was derived for a dilute ideal gas mixture, it has been often applied to condensed phase media [15].

The use of the Maxwell-Stefan relations is quite complicated because the diffusion velocities $\mathbf{V}_{i}$ are not expressed explicitly in terms of the concentration gradients. A common practice is to use the generalized Fick equations

$$
\mathbf{V}_{i}=\sum_{j} \mathbb{D}_{i j} \nabla X_{j}
$$


with coefficients $\mathbb{D}_{i j}$, referred to as Fick diffusivities, that are related to the binary diffusivities $\mathscr{D}_{i j}$, but unlike the binary diffusivities they are concentration dependent and may not necessarily be all positive. They must, however, satisfy the constraints [12]

$$
\mathbb{D}_{i j}=\mathbb{D}_{j i}, \quad \text { for all } i, j, \quad \sum_{i} \mathbb{D}_{i j} Y_{i}=0 \quad \text { for all } j .
$$

The expressions relating Fick and binary diffusivities for a ternary mixture [16] are listed in the Appendix. Converting eq. (21) from mole to mass fraction yields:

$$
Y_{i} V_{i}=a_{i} \nabla Y_{1}+b_{i} \nabla Y_{2}, \quad i=1,2
$$

where

$$
\begin{array}{r}
a_{1}=\frac{W_{1}\left(Y_{2}+Y_{3}\right)\left(-Y_{2} W_{3}-W_{2}+Y_{2} W_{2}\right) \mathscr{D}_{13} \mathscr{D}_{12}+Y_{1} Y_{2} W_{2}\left(W_{1}-W_{3}\right) \mathscr{D}_{23} \mathscr{D}_{12}}{Y_{1} W_{2} W_{3} \mathscr{D}_{23}+Y_{2} W_{1} W_{3} \mathscr{D}_{13}+Y_{3} W_{2} W_{1} \mathscr{D}_{12}} \\
-\frac{W_{3} Y_{1}\left(W_{2}-Y_{2} W_{2}+Y_{2} W_{1}\right) \mathscr{D}_{23} \mathscr{D}_{13}}{Y_{1} W_{2} W_{3} \mathscr{D}_{23}+Y_{2} W_{1} W_{3} \mathscr{D}_{13}+Y_{3} W_{2} W_{1} \mathscr{D}_{12}} \\
b_{1}=-\frac{Y_{1} W_{1}\left(Y_{2}+Y_{3}\right)\left(W_{2}-W_{3}\right) \mathscr{D}_{13} \mathscr{D}_{12}+Y_{1} W_{2}\left(-W_{1}+Y_{1} W_{1}-W_{3} Y_{1}\right) \mathscr{D}_{23} \mathscr{D}_{12}}{Y_{1} W_{2} W_{3} \mathscr{D}_{23}+Y_{2} W_{1} W_{3} \mathscr{D}_{13}+Y_{3} W_{2} W_{1} \mathscr{D}_{12}} \\
+\frac{Y_{1} W_{3}\left(-Y_{1} W_{2}-W_{1}+Y_{1} W_{1}\right) \mathscr{D}_{23} \mathscr{D}_{13}}{Y_{1} W_{2} W_{3} \mathscr{D}_{23}+Y_{2} W_{1} W_{3} \mathscr{D}_{13}+Y_{3} W_{2} W_{1} \mathscr{D}_{12}} \\
a_{2}=-\frac{Y_{2} W_{1}\left(-W_{2}+Y_{2} W_{2}-Y_{2} W_{3}\right) \mathscr{D}_{13} \mathscr{D}_{12}+Y_{2} W_{2}\left(Y_{1}+Y_{3}\right)\left(W_{1}-W_{3}\right) \mathscr{D}_{23} \mathscr{D}_{12}}{Y_{1} W_{2} W_{3} \mathscr{D}_{23}+Y_{2} W_{1} W_{3} \mathscr{D}_{13}+Y_{3} W_{2} W_{1} \mathscr{D}_{12}} \\
-\frac{Y_{2} W_{3}\left(-Y_{2} W_{2}+Y_{2} W_{1}+W_{2}\right) \mathscr{D}_{23} \mathscr{D}_{13}}{Y_{1} W_{2} W_{3} \mathscr{D}_{23}+Y_{2} W_{1} W_{3} \mathscr{D}_{13}+Y_{3} W_{2} W_{1} \mathscr{D}_{12}} \\
b_{2}=\frac{Y_{2} Y_{1} W_{1}\left(-W_{3}+W_{2}\right) \mathscr{D}_{13} \mathscr{D}_{12}}{+W_{2}\left(Y_{1}+Y_{3}\right)\left(-W_{1}+Y_{1} W_{1}-Y_{1} W_{3}\right) \mathscr{D}_{23} \mathscr{D}_{12}} \\
Y_{1} W_{2} W_{3} \mathscr{D}_{23} \\
+Y_{2} W_{1} W_{3} \mathscr{D}_{13}+Y_{3} W_{2} W_{1} \mathscr{D}_{12} \\
+\frac{Y_{2} W_{3}\left(-Y_{1} W_{2}-W_{1}+Y_{1} W_{1}\right) \mathscr{D}_{23} \mathscr{D}_{13}}{Y_{1} W_{2} W_{3} \mathscr{D}_{23}+Y_{2} W_{1} W_{3} \mathscr{D}_{13}+Y_{3} W_{2} W_{1} \mathscr{D}_{12}}
\end{array}
$$

The species equations (7)-(8) can then be written as

$$
\begin{aligned}
\rho u \frac{d Y_{1}}{d x}+\frac{d}{d x}\left[\rho\left(a_{1} \frac{d Y_{1}}{d x}+b_{1} \frac{d Y_{2}}{d x}\right)\right] & =-W_{1} \omega, \\
\rho u \frac{d Y_{2}}{d x}+\frac{d}{d x}\left[\rho\left(a_{2} \frac{d Y_{1}}{d x}+b_{2} \frac{d Y_{2}}{d x}\right)\right] & =-2 W_{2} \omega .
\end{aligned}
$$


A simplification that can be used for analytical convenience results from assuming equal molecular weights $W_{1}=$ $W_{2}=W_{3}$, then

$$
\begin{array}{r}
a_{1}=-\mathscr{D}_{13} \frac{\mathscr{D}_{12}+\left(\mathscr{D}_{23}-\mathscr{D}_{12}\right) Y_{1}}{\left(\mathscr{D}_{23}-\mathscr{D}_{12}\right) Y_{1}+\left(\mathscr{D}_{13}-\mathscr{D}_{12}\right) Y_{2}+\mathscr{D}_{12}} \\
b_{1}=\frac{\mathscr{D}_{23}\left(\mathscr{D}_{12}-\mathscr{D}_{13}\right) Y_{1}}{\left(\mathscr{D}_{23}-\mathscr{D}_{12}\right) Y_{1}+\left(\mathscr{D}_{13}-\mathscr{D}_{12}\right) Y_{2}+\mathscr{D}_{12}} \\
a_{2}=\frac{\mathscr{D}_{13}\left(\mathscr{D}_{12}-\mathscr{D}_{23}\right) Y_{2}}{\left(\mathscr{D}_{23}-\mathscr{D}_{12}\right) Y_{1}+\left(\mathscr{D}_{13}-\mathscr{D}_{12}\right) Y_{2}+\mathscr{D}_{12}} \\
b_{2}=-\mathscr{D}_{23} \frac{\mathscr{D}_{12}+\left(\mathscr{D}_{13}-\mathscr{D}_{12}\right) Y_{2}}{\left(\mathscr{D}_{23}-\mathscr{D}_{12}\right) Y_{1}+\left(\mathscr{D}_{13}-\mathscr{D}_{12}\right) Y_{2}+\mathscr{D}_{12}}
\end{array}
$$

\section{Asymptotic Solution - the Burke-Schumann limit}

We first present analytical results in the limit of infinitely fast chemical reaction, known in the literature as the Burke-Schumann limit. The solutions obtained in this limit provide a simple illustration of the flame structure. An appropriate time scale may be defined as the ratio of a representative length associated with the distance between particles and a characteristic microscale velocity associated with material deformation. The inverse of this time is the characteristic strain rate, and the fast chemistry limit corresponds to weak strain rates. The corresponding Damköhler number, or the ratio of the flow-to-chemistry time scales, is therefore large. Although asymptotic methods that accounts for finite rate chemistry and thus span a wider range of strain rates (up to flame extinction) are available for the related gaseous problem [17, 18], their extension to energetic materials is nontrivial and will be discussed in a future publication. Here we rely on numerical methods to examine the dependence of the solution on the strain rate for steady combustion.

In the fast chemistry limit the chemical reaction occurs along a sheet, at $x=x_{f}$ say, where the two reactants are in contact. Elsewhere, the chemical reaction is negligibly small and we are left solving the energy and species equations on either side of the sheet, with $\omega=0$. The flame sheet separates a region where there is only titanium $\left(x<x_{f}\right)$, from a region where there is only boron $\left(x>x_{f}\right)$. Since $Y_{2}=0$ for $x<x_{f}$, we find that

$$
\begin{array}{ll}
a_{1}=-\mathscr{D}_{13}, & b_{1}=\frac{\mathscr{D}_{23}\left(\mathscr{D}_{12}-\mathscr{D}_{13}\right) Y_{1}}{\mathscr{D}_{23} Y_{1}+\mathscr{D}_{12}\left(1-Y_{1}\right)}, \\
a_{2}=0, & b_{2}=-\frac{\mathscr{D}_{23} \mathscr{D}_{12}}{\mathscr{D}_{23} Y_{1}+\mathscr{D}_{12}\left(1-Y_{1}\right)} .
\end{array}
$$


and, since $Y_{1}=0$ for $x>x_{f}$, we find that

$$
\begin{aligned}
a_{1} & =-\frac{\mathscr{D}_{13} \mathscr{D}_{12}}{\mathscr{D}_{13} Y_{2}+\mathscr{D}_{12}\left(1-Y_{2}\right)}, & b_{1} & =0, \\
a_{2} & =\frac{\mathscr{D}_{13}\left(\mathscr{D}_{12}-\mathscr{D}_{23}\right) Y_{2}}{\mathscr{D}_{13} Y_{2}+\mathscr{D}_{12}\left(1-Y_{2}\right)}, & b_{2} & =-\mathscr{D}_{23} .
\end{aligned}
$$

We note parenthetically that the simplification of the coefficients $a_{i}, b_{i}$ applies even for unequal molecular weights. All variables must be continuous at the flame sheet, but their derivatives are not; the mass and energy fluxes must satisfy the relations

$$
\frac{\alpha}{Q / c_{p}}\left[\frac{d T}{d x}\right]=\frac{1}{W_{1}}\left[a_{1} \frac{d Y_{1}}{d x}+b_{1} \frac{d Y_{2}}{d x}\right]=\frac{1}{2 W_{2}}\left[a_{2} \frac{d Y_{1}}{d x}+b_{2} \frac{d Y_{2}}{d x}\right],
$$

where the square brackets $[\cdot]$ denote the jump, namely the difference between the values at $x_{f}^{+}$and $x_{f}^{-}$, and $\alpha=k / \rho c_{p}$ is the thermal diffusivity of the mixture. The conditions (25) imply that the fluxes of titanium and boron towards the flame sheet are in stoichiometric proportions, and they specify the proportion of heat from the total heat released conducted to one or the other side of the sheet.

For simplicity, we have also adopted in this section the constant density approximation. The mathematical problem on either side of the flame sheet then consists of

$$
\begin{array}{cc}
2 \epsilon x \frac{d T}{d x}+\alpha \frac{d^{2} T}{d x^{2}}=0 & \text { for } x \lessgtr x_{f} \\
2 \epsilon x \frac{d Y_{1}}{d x}+\mathscr{D}_{13} \frac{d^{2} Y_{1}}{d x^{2}}=0 & \text { for } x<x_{f} \\
Y_{1} \equiv 0 & \text { for } x>x_{f} \\
Y_{2} \equiv 0 & \text { for } x<x_{f} \\
2 \epsilon x \frac{d Y_{2}}{d x}+\mathscr{D}_{23} \frac{d^{2} Y_{2}}{d x^{2}}=0 & \text { for } x>x_{f}
\end{array}
$$

together with the boundary conditions (16)-(17), with $\rho$ assumed constant, and the jump relations

$$
\begin{gathered}
{[T]=\left[Y_{1}\right]=\left[Y_{2}\right]=0} \\
\frac{\alpha}{Q / c_{p}}\left[\frac{d T}{d x}\right]=-\frac{\mathscr{D}_{13}}{W_{1}}\left[\frac{d Y_{1}}{d x}\right]=-\frac{\mathscr{D}_{23}}{2 W_{2}}\left[\frac{d Y_{2}}{d x}\right] .
\end{gathered}
$$


across $x=x_{f}$. The solution is readily obtained as

$$
\begin{gathered}
T= \begin{cases}T_{\infty}+\left(T_{f}-T_{\infty}\right) \frac{1+\operatorname{erf}(\sqrt{\epsilon / \alpha} x)}{1+\operatorname{erf}\left(\sqrt{\epsilon / \alpha} x_{f}\right)} & x<x_{f} \\
T_{\infty}+\left(T_{f}-T_{\infty}\right) \frac{1-\operatorname{erf}(\sqrt{\epsilon / \alpha} x)}{1-\operatorname{erf}\left(\sqrt{\epsilon / \alpha} x_{f}\right)} & x>x_{f}\end{cases} \\
Y_{1}= \begin{cases}1-\frac{1+\operatorname{erf}\left(\sqrt{\epsilon / \mathscr{D}_{13}} x\right)}{1+\operatorname{erf}\left(\sqrt{\epsilon / \mathscr{D}_{13}} x_{f}\right)} & x<x_{f} \\
0 & x>x_{f}\end{cases} \\
Y_{2}= \begin{cases}0 & x<x_{f} \\
1-\frac{1-\operatorname{erf}\left(\sqrt{\epsilon / \mathscr{D}_{23}} x\right)}{1-\operatorname{erf}\left(\sqrt{\epsilon / \mathscr{D}_{23}} x_{f}\right)} & x>x_{f}\end{cases}
\end{gathered}
$$

The position $x_{f}$ of the flame sheet and the adiabatic flame temperature $T_{f}$, defined as the value of $T$ at the flame sheet, satisfy

$$
\begin{gathered}
\frac{1+\operatorname{erf}\left(\sqrt{\epsilon / \mathscr{D}_{13}} x_{f}\right)}{1-\operatorname{erf}\left(\sqrt{\epsilon / \mathscr{D}_{23}} x_{f}\right)}=v \sqrt{\frac{\mathscr{D}_{13}}{\mathscr{D}_{23}}} \frac{e^{\epsilon x_{f}^{2} / \mathscr{D}_{23}}}{e^{\epsilon x_{f}^{2} / \mathscr{D}_{13}}} \\
T_{f}=T_{\infty}+\frac{1}{2} \frac{Q / c_{p}}{W_{1}} \sqrt{\frac{\mathscr{D}_{13}}{\alpha}} \frac{1-\operatorname{erf}^{2}\left(\sqrt{\epsilon / \alpha} x_{f}\right)}{1+\operatorname{erf}\left(\sqrt{\epsilon / \mathscr{D}_{13}} x_{f}\right)} \frac{e^{\epsilon x_{f}^{2} / \alpha}}{e^{\epsilon x_{f}^{2} / \mathscr{D}_{13}}}
\end{gathered}
$$

where $v=2 W_{2} / W_{1}$ is a mass-weighted stoichiometric ratio. The position $x_{f}$ is determined from the transcendental equation (33) using an iterative process. We note that for a given strain rate $\epsilon$ the position $x_{f}$ depends only on the binary diffusivities $\mathrm{Ti}^{-} \mathrm{TiB}_{2}$ and $\mathrm{B}-\mathrm{TiB}_{2}$, and is independent of the diffusivity of Ti-B because there is no boron in the titanium region and vice-versa. Once $x_{f}$ is determined, the flame temperature can be calculated from (34) by direct evaluation. Evidently, the latter depends on the heat released $Q$ and thermal diffusivity $\alpha$.

For equal diffusivities $\mathscr{D}_{13}=\mathscr{D}_{23} \equiv \mathscr{D}$, eq. (33) reduces to

$$
\operatorname{erf}\left(\sqrt{\epsilon / \mathscr{D}} x_{f}\right)=\frac{v-1}{v+1}
$$

For the titanium-boron reaction the mass-weighted stoichiometric ratio $v \approx 0.45$, implying that $x_{f} \approx-0.41 \sqrt{\mathscr{D} / \epsilon}$ and the flame sheet lies on the titanium side of the stagnation plane. If the Lewis number is assumed equal to one, i.e., $\mathscr{D}=\alpha$, the flame temperature is given by

$$
T_{f}=T_{\infty}+\frac{Q / c_{p} W_{1}}{1+v} .
$$


In the absence of differential (unity Lewis number) and preferential (unequal mass diffusivities) diffusion, the flame temperature results from a simple energy balance.

Figure 2 displays the dependence of the scaled flame sheet location on the ratio $\mathscr{D}_{23} / \mathscr{D}_{13}$, for a fixed $v$. This ratio is typically larger than one, since boron atoms have an effectively smaller atomic radius and hence diffuse more readily through titanium-diboride than does the titanium atom $[19,20]$. We see that the flame sheet generally resides on the titanium side of the stagnation plane and moves further to the left when the diffusivity of boron into the titaniumdiboride products increases relative to the diffusivity of titanium into the titanium-diboride products.

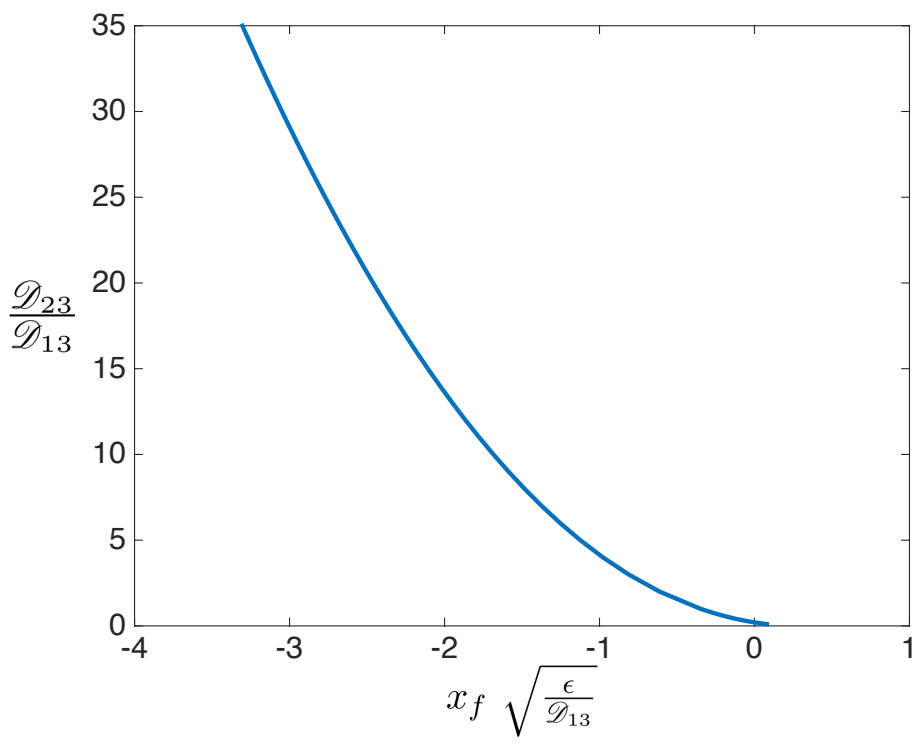

Figure 2. Variation of the flame sheet position with $\mathscr{D}_{23} / \mathscr{D}_{13}$, based on the asymptotic solution

The only explicit results presented here from the asymptotic solution are the expressions for the flame position and temperature. Predictions based on these expressions are used in conjunction with experimental data to extract kinetic parameters that are not well known or were measured under limited conditions. Moreover, the asymptotic solution is used to validate the numerical solution of the governing equations for large values of the Damköhler number (or small strain rates) and to properly calibrate the strain rate, in order to derive extinction conditions. 


\section{Numerical solution - finite-rate chemistry}

To examine the effects of finite-rate chemistry, the boundary value problem consisting of equations (4)-(6) and (23)-(24) and boundary conditions (15)-(17) must be addressed. The numerical procedure is described next, followed by results pertaining to titanium-boron combustion.

\subsection{Numerical Procedure}

Steady solutions of the aforementioned boundary value problem are obtained numerically as the long time behavior of the time-dependent equations

$$
\begin{gathered}
\rho \frac{\partial \bar{v}}{\partial t}+\rho u \frac{\partial \bar{v}}{\partial x}+\rho \bar{v}^{2}=C \\
\rho \frac{\partial Y_{1}}{\partial t}+\rho u \frac{\partial Y_{1}}{\partial x}+\frac{\partial}{\partial x}\left[\rho\left(a_{1} \frac{\partial Y_{1}}{\partial x}+b_{1} \frac{\partial Y_{2}}{\partial x}\right)\right]=-W_{1} \omega \\
\rho \frac{\partial Y_{2}}{\partial t}+\rho u \frac{\partial Y_{2}}{\partial x}+\frac{\partial}{\partial x}\left[\rho\left(a_{2} \frac{\partial Y_{1}}{\partial x}+b_{2} \frac{\partial Y_{2}}{\partial x}\right)\right]=-2 W_{2} \omega \\
\rho c_{p}\left(\frac{\partial T}{\partial t}+u \frac{\partial T}{\partial x}\right)-k \frac{\partial^{2} T}{\partial x^{2}}=Q \omega,
\end{gathered}
$$

with $\bar{v}$ obtained from

$$
\rho \bar{v}=-\frac{d}{d x}(\rho u)
$$

and $\rho$ from (14). Initially, these equations were solved using an explicit time marching method until the solution converges to its equilibrium state. The integration in time starts with an initial guess, taken here as the asymptotic solution discussed in the previous section. A fourth order approximation was used to compute the first order and second order space derivatives and a fourth order Runge-Kutta (RK4) method was used for time stepping. The extent of the numerical domain depends on the strain rate value, with lower strain rates requiring a larger domain. This, however, can be overcome by normalizing $x$ with the thermal diffusion length $l_{d}=\sqrt{\alpha / \epsilon}$, as is also evident from the analytical form of the asymptotic solution. Due to large stiffness arising from the Arrhenius exponential in the reaction rate term, we found that the time step, in general, could not exceed $10^{-9}$ and to properly describe the solution at low strain rates where the reaction zone becomes extremely thin, a fine grid is also required. As a result, convergence to the equilibrium state was very slow even after parallelizing the numerical code. Determining the solution over a wide range of strain rate conditions requires a faster converging algorithm. 
To overcome the computational stiffness, an implicit time relaxation method was implemented. First and second order spatial derivatives are approximated by second order finite difference schemes on a uniform grid, and for time stepping we use a backward Euler method, such that a generic equation of the form $\partial \phi / \partial t=f(t, \phi)$ is approximated by $\phi^{n+1}=\phi^{n}+\Delta t f\left(t^{n+1}, \phi^{n+1}\right)$, where $n$ denotes the time step and $\Delta t$ the time increment. A damped Newton-Raphson solver is then used for the solution of the resulting nonlinear system at each time step. The stiffness in the governing equations lead to strong fluctuations, and consequently sharp gradients in the Jacobian matrix that may cause the numerical solution to diverge. We have therefore implemented a relaxation parameter during each iterative update to limit the fluctuations; the value 0.1 was found appropriate for our calculations. The PetSC sparse solver [21] was used for the system that arises during the Newton-Raphson iteration. This approach has significantly reduced the time step relative to the explicit scheme from $10^{-9}$ to $10^{-2}$, for the same spatial grid distribution.

In order to generate solutions for increasing values of the strain rate $\epsilon$ and draw response curves of quantities of interest (e.g., flame temperature, mass fraction of unconsumed reactants, etc.) as a function of $\epsilon$, we start with a small strain rate value of $\epsilon=0.01 \mathrm{~s}^{-1}$ using the asymptotic solution as an initial guess, and advance in time until the incremental changes in the solution at all points in the domain of integration are less than a tolerance error, here taken as $10^{-3}$. Convergence is guaranteed only when the long-time equilibrium solution is stable. This approach, therefore, works well for small-to-moderate values of $\epsilon$, but fails at larger values when the solution of the boundary value problem becomes multi-valued, and the response curve develops a turning point with stable and unstable branches. To construct all steady solutions, stable and unstable, particularly near the turning point, we use the continuation approach proposed by Kurdyumov and Matalon [22]. The time-dependent equations (35)-(39) were solved with an additional constraint that the temperature remains constant at some reference point, say $T\left(x^{*}\right)=T^{*}$. The constraint is used to iterate on the value of $\epsilon$ until both, the strain rate and the space distribution of solution, do not vary significantly from one time step to the next. By selecting $T^{*}$ judiciously, this procedure always converges allowing to generate the entire response curve through a turning point. When computing steady states that are expected to be unstable, the time steps must be adaptively changed while keeping the damped newton iterations. It is crucial to start with small time steps, $10^{-6}$ say, and gradually increase $\Delta t$ to $10^{-2}$. Finally, we note that the time $t$ is used in this context only as an iterative parameter. 


\subsection{Physical parameters}

Table 1 lists representative values of physical parameters based on a literature survey; some values required by the model are easier to estimate than others and are obtained from standard thermal property measurements. The table lists values for the constant pressure heat capacity, $c_{p}$, and thermal conductivity, $k$, that represent averaged values for the mixture. However, the determination of the binary mass diffusivities is more problematic. Experimental values for $\mathscr{D}_{13}$ and $\mathscr{D}_{23}$ have been reported $[19,20]$, but not at conditions that are present in the reaction zone. Better estimates could be obtained from our derived asymptotic expressions, when used in conjunction with experimentally measured flame temperatures.

Trunov et al. [13] measured adiabatic flame temperatures for the titanium-boron reaction of approximately 2400-

\begin{tabular}{|l|c|c|c|}
\hline Property & Symbol & Value & Reference \\
\hline \hline Heat release & $Q$ & $-323.8 \mathrm{~kJ} / \mathrm{mol}$ & {$[23]$} \\
\hline Molar mass of Ti & $W_{1}$ & $47.87 \mathrm{~g} / \mathrm{mol}$ & \\
\hline Molar mass of B & $W_{2}$ & $10.81 \mathrm{~g} / \mathrm{mol}$ & \\
\hline Molar mass of TiB ${ }_{2}$ & $W_{3}$ & $69.85 \mathrm{~g} / \mathrm{mol}$ & \\
\hline Averaged molar mass & $W_{c}$ & $50 \mathrm{~g} / \mathrm{mol}$ & \\
\hline Intrinsic density of Ti & $\hat{\rho}_{1_{0}}$ & $4.5 \mathrm{~g} / \mathrm{cc}$ & \\
\hline Intrinsic density of B & $\hat{\rho}_{2_{0}}$ & $2.34 \mathrm{~g} / \mathrm{cc}$ & {$[27]$} \\
\hline Intrinsic density of TiB 2 & $\hat{\rho}_{3_{0}}$ & $4.52 \mathrm{~g} / \mathrm{cc}$ & \\
\hline Averaged density & $\hat{\rho}_{c_{0}}$ & $3.8 \mathrm{~g} / \mathrm{cc}$ & \\
\hline Heat capacity & $c_{p}$ & $900 \mathrm{~J} /(\mathrm{kg} \mathrm{K})$ & {$[23]$} \\
\hline Thermal conductivity & $k$ & $36 \mathrm{~W} /(\mathrm{m} \mathrm{K})$ & {$[24,25,26]$} \\
\hline Pre exponential factor & $\mathcal{B}$ & $7.6 \mathrm{e} 16 \mathrm{~mol} /\left(\mathrm{m}^{3} \mathrm{~s}\right)$ & {$[27]$} \\
\hline Activation energy & $E_{a}$ & $318 \mathrm{~kJ} / \mathrm{mol}$ & \\
\hline Binary diffusivity of Ti-B & $\mathscr{D}_{12}$ & $0.2 \mathrm{~m} / \mathrm{s}$ & \\
\hline
\end{tabular}

Table 1. Property values of representative physical parameters used in the computations 


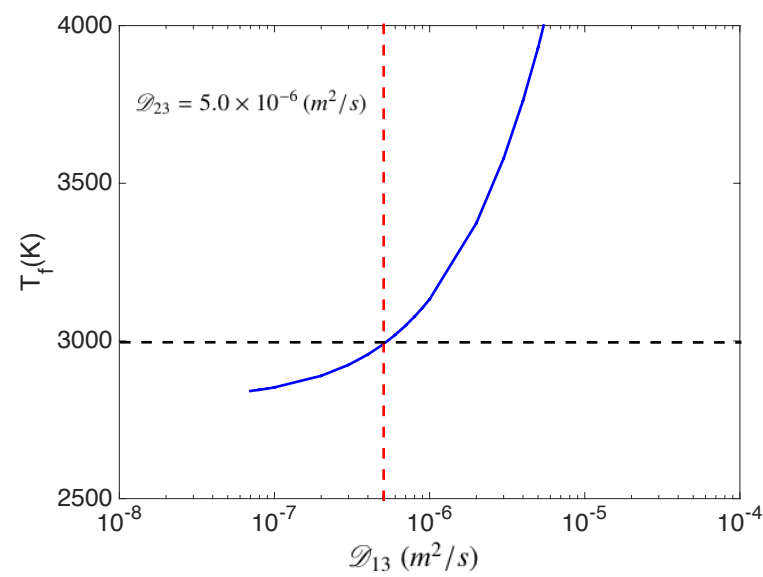

(a)

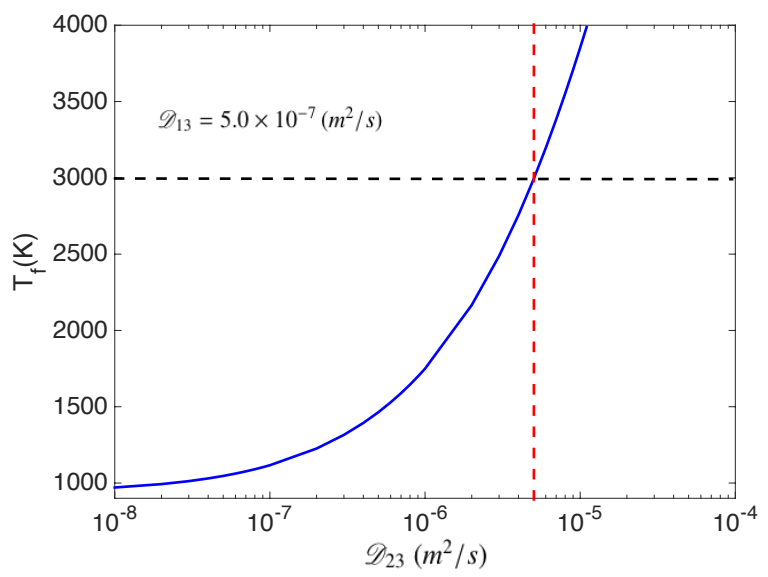

(b)

Figure 3. Variation of the adiabatic flame temperature $T_{f}$ with (a) variations in $\mathscr{D}_{13}$, for given $\mathscr{D}_{23}=5.0 \cdot 10^{-6} \mathrm{~m}^{2} / \mathrm{s}$, and (b) variations in $\mathscr{D}_{23}$, for given $\mathscr{D}_{13}=5.0 \cdot 10^{-7} \mathrm{~m}^{2} / \mathrm{s}$, relative to base-line values marked by a dashed line.

$3300 \mathrm{~K}$. One expects, similar to the values measured experimentally at low temperatures [20], that the binary diffusivities of both, boron and titanium into titanium-diboride, are significantly smaller than the mixture thermal diffusivity, and that $\mathscr{D}_{23} \gg \mathscr{D}_{13}$. Taking $\mathscr{D}_{23} / \mathscr{D}_{13}=10$ as a base-line model value, and the estimate $T_{f}=3000 \mathrm{~K}$ for the flamesheet temperature, our formulas (33)-(34) yield $\mathscr{D}_{13} \approx 5 \times 10^{-7} \mathrm{~m}^{2} / \mathrm{s}$ and $\mathscr{D}_{23} \approx 5 \times 10^{-6} \mathrm{~m}^{2} / \mathrm{s}$. Figure 3 shows changes in flame temperature as a result of variations in the binary diffusivities $\mathscr{D}_{13}$ and $\mathscr{D}_{23}$ relative to the base-line values, for the fixed ratio of $\mathscr{D}_{23} / \mathscr{D}_{13}$.

\subsection{Low strain rates}

We start by presenting results pertaining to low strain rates, where the solution can be compared directly to the asymptotic solution discussed above. Since the latter was obtained under the constant density assumption, the average value $\rho=3.8 \mathrm{~g} / \mathrm{cc}$ was selected, abandoning the equation of state (14). The expressions for the diffusion coefficients were used without resorting to the approximation of equal molecular weights, since the reduced form of these relations led to nearly identical results.

Figures 4(a)-(b) show a comparison of the temperature and mass fraction profiles between the computed solution for $\epsilon=0.01 \mathrm{~s}^{-1}$ and the corresponding asymptotic expressions. The spatial coordinate is normalized with the thermal 


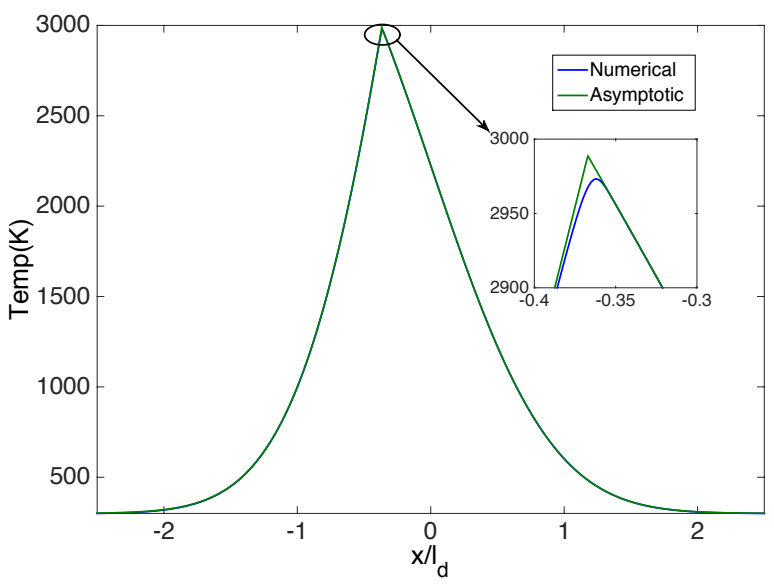

(a) Temperature profile

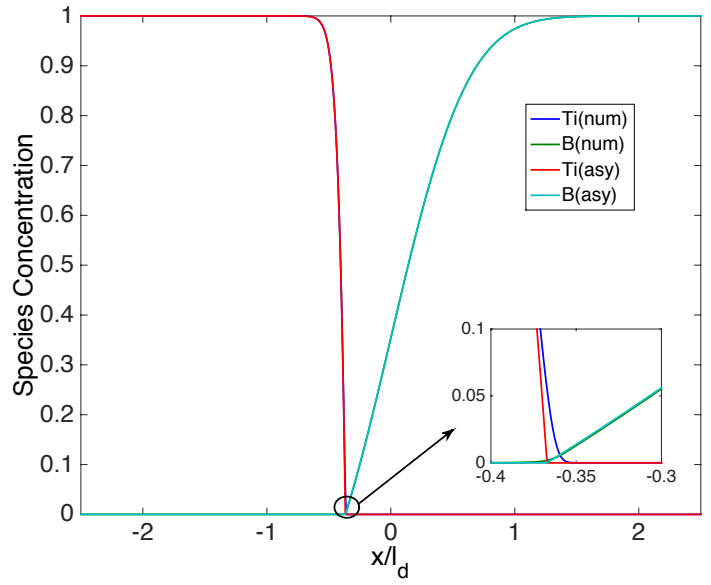

(b) Mass fractions profiles

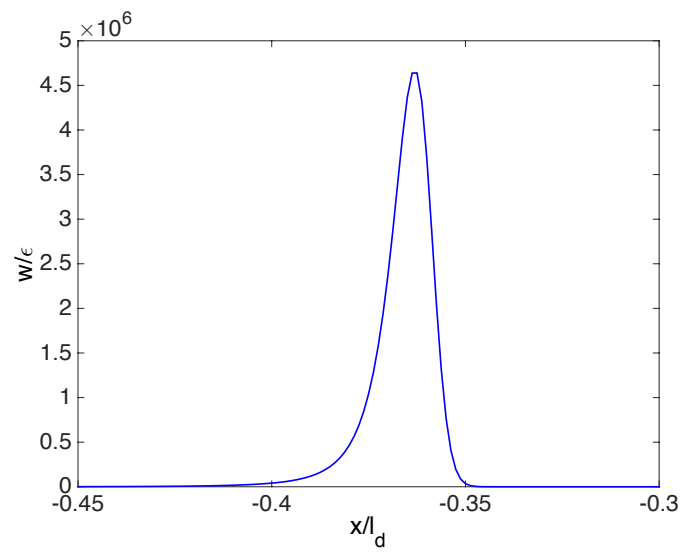

(c) Reaction rate

Figure 4. Comparison between the numerical solution and asymptotic solutions for a small strain rate value $\epsilon=0.01 s^{-1}$

diffusion length $l_{d}=\sqrt{\alpha / \epsilon}=3 \cdot 2410^{-4} \mathrm{~cm}$. For such low values of strain rate combustion is nearly complete, with both reactants consumed in a very thin reaction zone. There is excellent agreement between the computed and asymptotic profiles except for very small changes near the reaction zone, as shown in the figure inserts. Evidently, for finite $\epsilon$ however small, the reaction zone has a finite thickness, which is adequately captured by the numerical solution as shown in Fig. 4(c). The excellent agreement between the numerical and asymptotic solutions also serves as a validation of our numerical methodology that properly resolves the stiffness in the governing equations arising from the exponential Arrhenius term. 


\subsection{Moderate strain rates - constant density}

Next we consider the entire response of the flame to increasing strain rates, from complete combustion corresponding to low strain rates to flame extinction occurring at significantly higher values of $\epsilon$.

We first examine the solution using two diffusion formulations: the complete expressions for the diffusion coefficients $a_{i}, b_{i}$, and the simplified form resulting from equal molecular weights. We note that the general diffusion expressions add nonlinearity to an already stiff problem. Therefore, to facilitate the computations we have retained the constant-density approximation in the results presented in this subsection, which effectively decouples the flow and combustion fields; variable density solutions will be presented in the next subsection. Figures 5 and 6 show response curves of various flame characteristics to the imposed strain rate $\epsilon$, based on the two diffusion formulations. These include the flame position $x_{f}$ (defined as the location where the temperature reaches its maximum value), the flame temperature $T_{f}$ (the value of $T$ at $x_{f}$ ), and the mass fractions of unconsumed titanium and boron (the values of $Y_{1}$ and $Y_{2}$ evaluated at $x_{f}$ ). We note that the precise evaluation of the flame position, and the temperature and mass fractions evaluated at this location, depend on the step size used in the computations and on the value of the thermal diffusion length $l_{d}$, which decreases from $3.24 \cdot 10^{-5} \mathrm{~cm}$ to $1.3 \cdot 10^{-6} \mathrm{~cm}$ as the strain rate increases from $\epsilon=0.01 \mathrm{~s}^{-1}$ to $\epsilon=6.5 \mathrm{~s}^{-1}$ (which is very near extinction). A Matlab Curve Fit [29] tool was used to fit the numerical data to a smoothing spline, which was then used for the determination of $x_{f}$ and for evaluating all properties at this location. The same tool was used to fit smoothing spline curves presented in all the figures, to the discrete data obtained numerically.

From the response curves of Figs. 5-6 the following physical picture emerges. Since at low strain rates the chemical reaction time is much shorter than the flow time, the reaction proceeds immediately as titanium and boron get in contact. The reaction occurs in a very thin zone (or a sheet) where both reactants are completely consumed. The flame lies on the titanium side $(x<0)$ and the flame temperature reaches its maximum value. Upon increasing the strain rate, the flow time relative to the chemical reaction time is shortened and a fraction of titanium and boron escape mixing and leaks through the reaction zone. As a result of incomplete combustion, the flame moves towards the boron side and the flame temperature drops. The relatively larger leakage of titanium as opposed to boron stems from the fact that $\mathscr{D}_{13} \ll \mathscr{D}_{23}$, which implies much larger fluxes of boron towards the reaction zone and consequently a more 


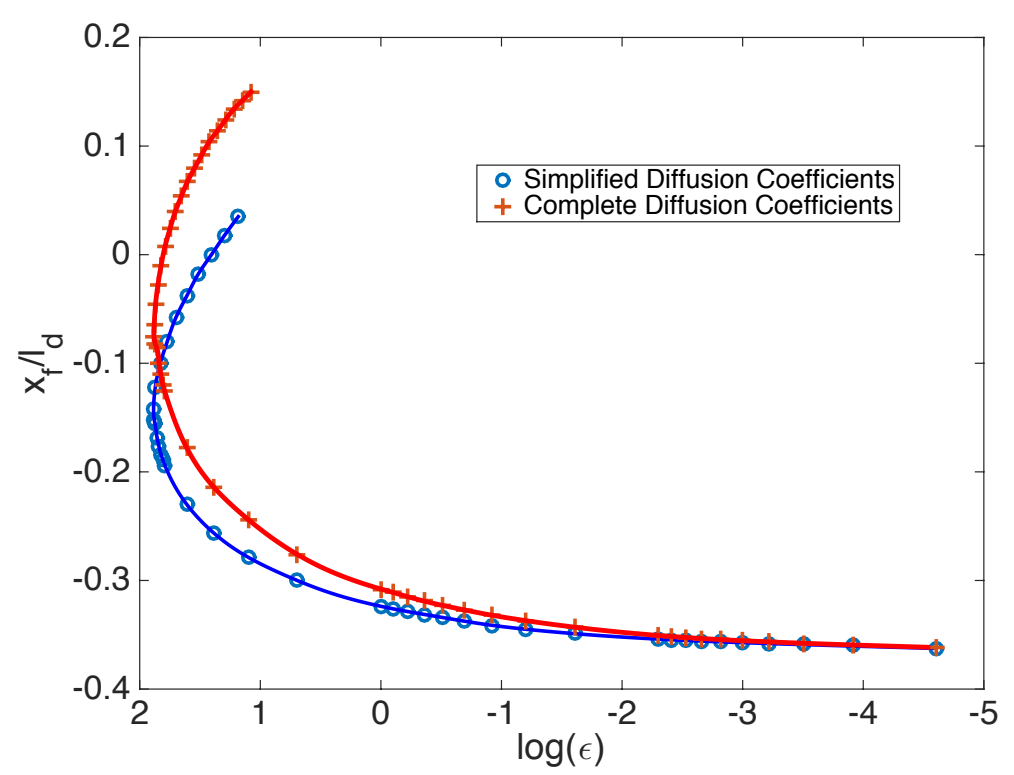

(a) Flame position

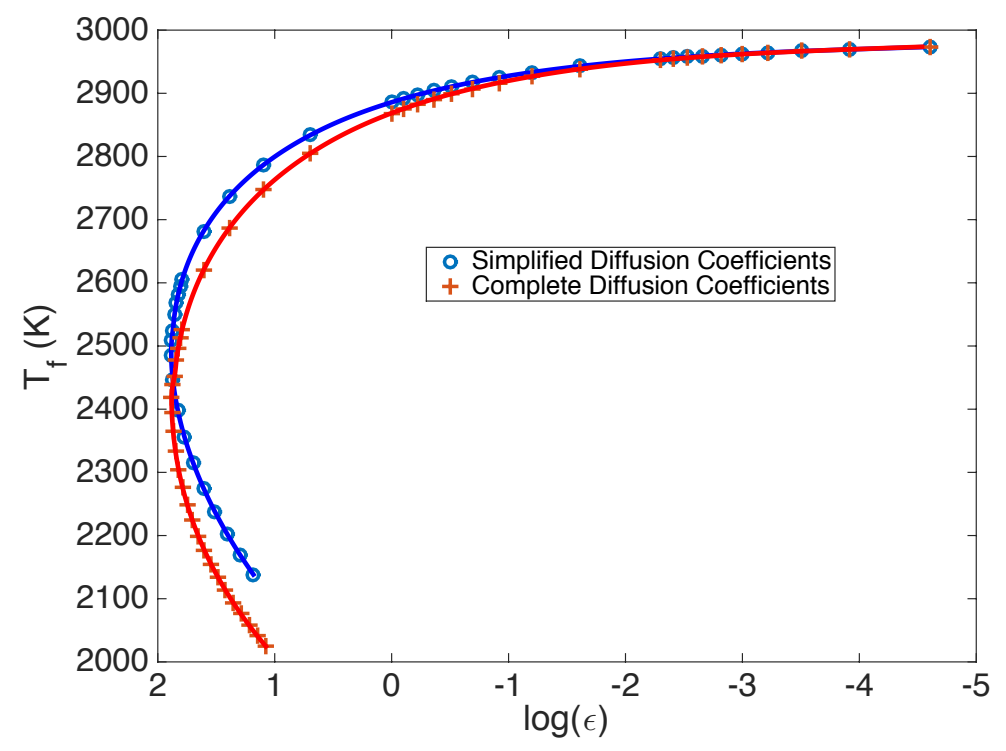

(b) Flame temperature

Figure 5. Response curves of flame position and flame temperature versus strain rate. The two curves (red/blue) correspond to the complete and simplified diffusion formulations.

complete consumption of boron. This trend continues until the fraction of unconsumed reactants exceeds a critical threshold and the flame temperature drops significantly from its adiabatic value. For larger values of the strain rate steady burning is no longer possible. The critical state, represented by the turning point on the response curves and 


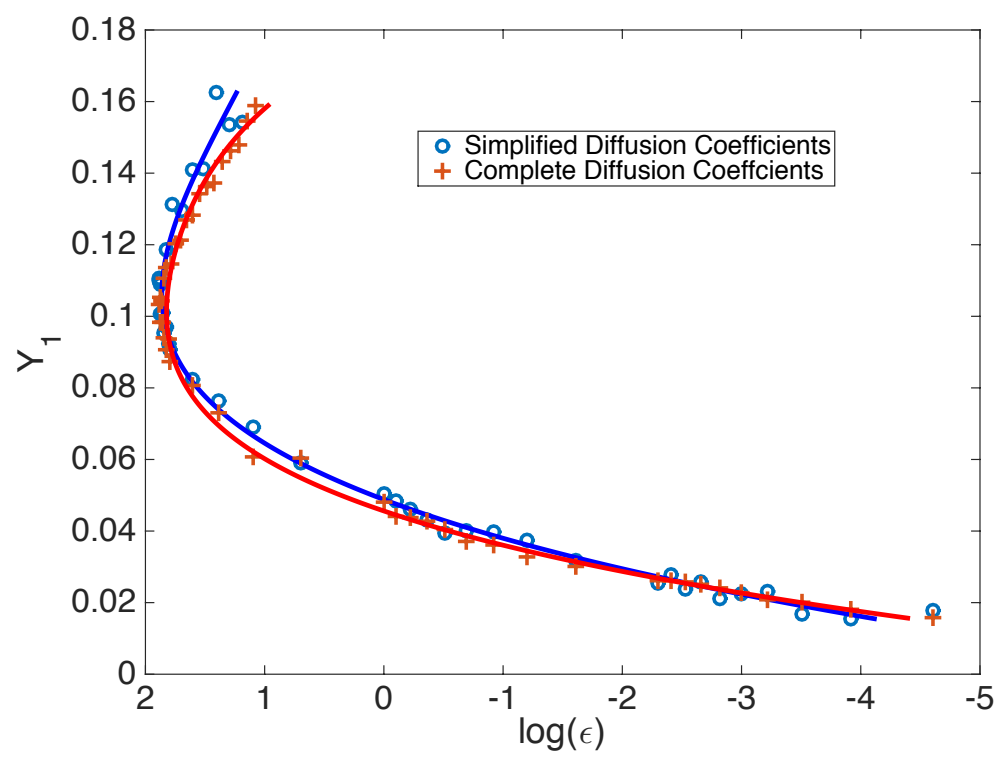

(a) Unconsumed titanium mass fraction

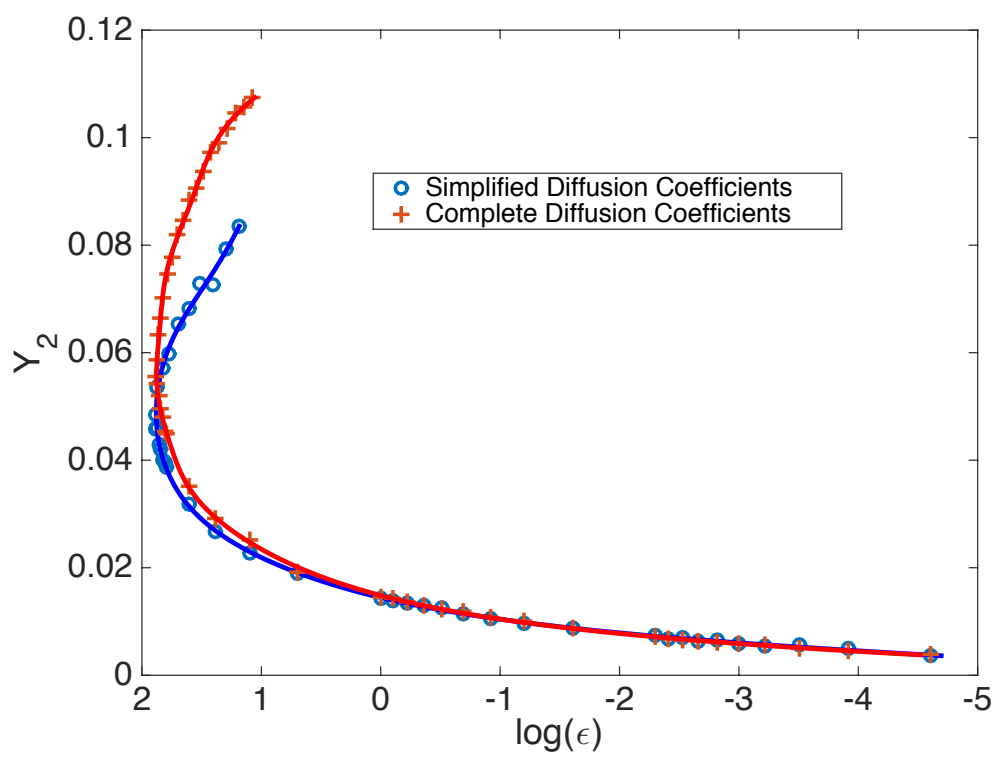

(b) Unconsumed boron mass fraction 
The two diffusion formulations lead to identical results at low strain rates and predict the exact same extinction strain rates. There are small, insignificant differences in flame temperature at and near extinction, which can be traced to the slight difference in flame location. Being influenced primarily by the binary diffusivity $\mathscr{D}_{13} \ll \mathscr{D}_{23}$, the simplified diffusion formulation predicts a flame position that is slightly tilted towards the titanium $(x<0)$ side. Due to the negligible difference between the two formulations, the simplified diffusion formulation will be used in the following section in order to save computational time.

\subsection{Moderate strain rates - variable density}

When variations in density are accounted for, the flow field no longer satisfies (19) and must be obtained by solving the momentum equation (5) with $\rho$ given by (14). The boundary conditions as $x \rightarrow \pm \infty$ imply that $\bar{v}$ tends to a constant on either end, with the asymptotes subject to the constraint (9). Figure 7 shows profiles of $\bar{v}$ computed for two values of $\epsilon$; the low strain rate value corresponding to conditions close to the Burke-Schumann solution and the larger strain rate value corresponding to near-extinction conditions. Variations in $\bar{v}$ across the field are due to density changes, which are more pronounced in the combustion region $-0.5 \lesssim x / 1_{D} \lesssim 0.5$.

Density profiles are shown in Figure 8 for the same two values of $\epsilon$. Generally, the density would vary from

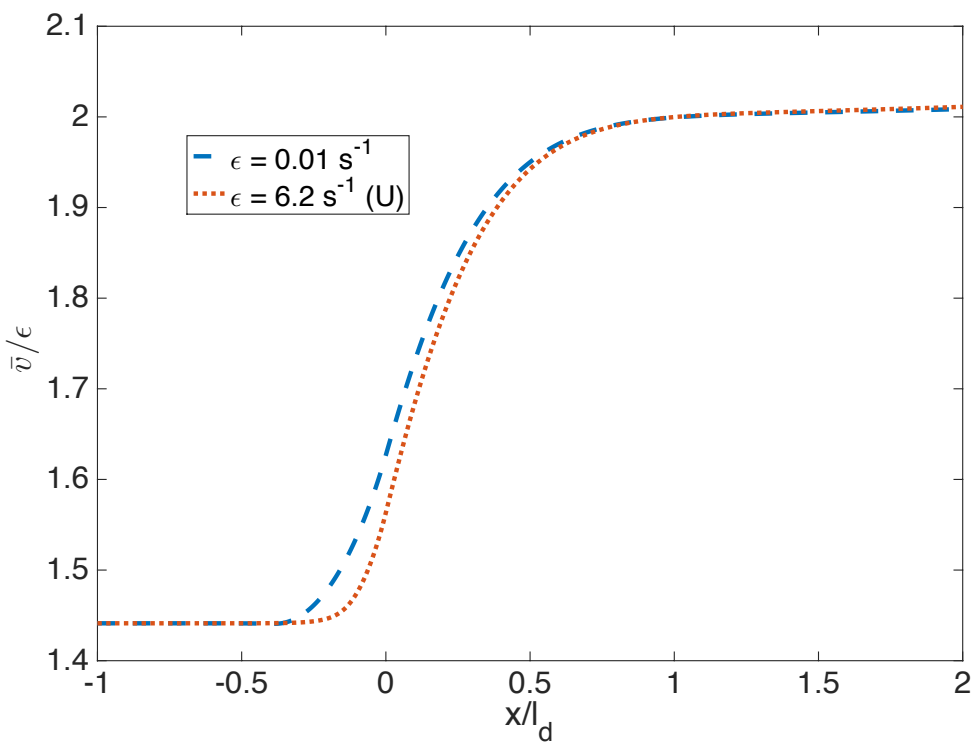

Figure 7. Profiles of the transverse velocity $\bar{v}$ across the combustion zone for two strain rate values. 


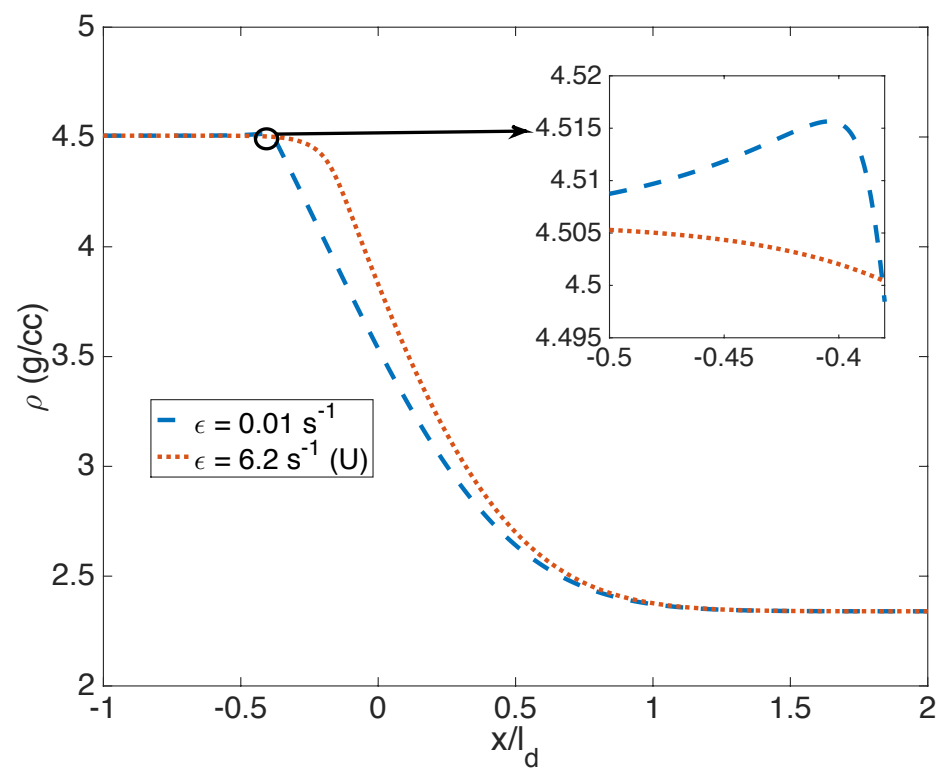

Figure 8. Density profiles across the combustion zone for two strain rate values.

$\hat{\rho}_{1_{0}}=4.5 \mathrm{~g} / \mathrm{cc}$ on the titanium side to $\hat{\rho}_{2_{0}}=2.34 \mathrm{~g} / \mathrm{cc}$ on the boron side. Since to the density of $\mathrm{TiB}_{2}$ is slightly higher than that of $\mathrm{Ti}$, there is a noticeable jump in density near the reaction zone at low strain rates (see the figure inset), where the reaction is more vigorous and the production of $\mathrm{TiB}_{2}$ higher. This increase in density diminishes at higher strain rates due to lower $\mathrm{TiB}_{2}$ production and increase of titanium leakage through the reaction zone.

Response curves of flame position and temperature vs strain rates, for constant and variable density conditions, are shown in Fig. 9. The flame temperature $T_{f}$ and flame position $x_{f}$ are consistently higher for the constant density case than for the variable density case, for all values of $\epsilon$. The difference is very small and is of no physical significance, being primarily due to the selected mean density value adopted in the constant density formulation. The small difference between the two solutions indicates that the composition distribution has little effect on the overall density, for practically all strain rate values. The flame temperature at extinction is approximately $2500 \mathrm{~K}$, corresponding to a drop in approximately $500 \mathrm{~K}$ from the adiabatic flame temperature $T_{f}=2950 \mathrm{~K}$ (or $3000 \mathrm{~K}$ for the constant density case). The flame position in the Burke-Schumann limit is determined from the condition that the two separate reactants meet in stoichiometric proportions, with the temperature playing no role in this balance. One may therefore attribute the slight variation in flame position between the constant and variable cases to the dependence of species distribution on the overall density. As with the flame temperature, the predicted values of $x_{f}$ at extinction by the two formulations is 
very close, with the difference being less than $0.1 \%$.

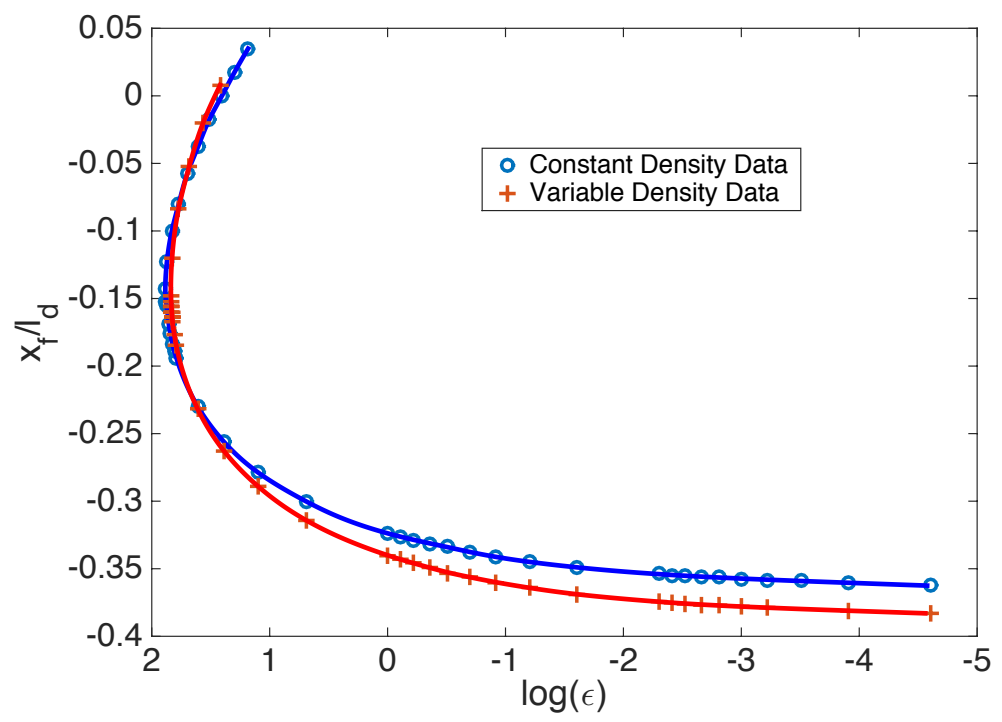

(a) flame psotition

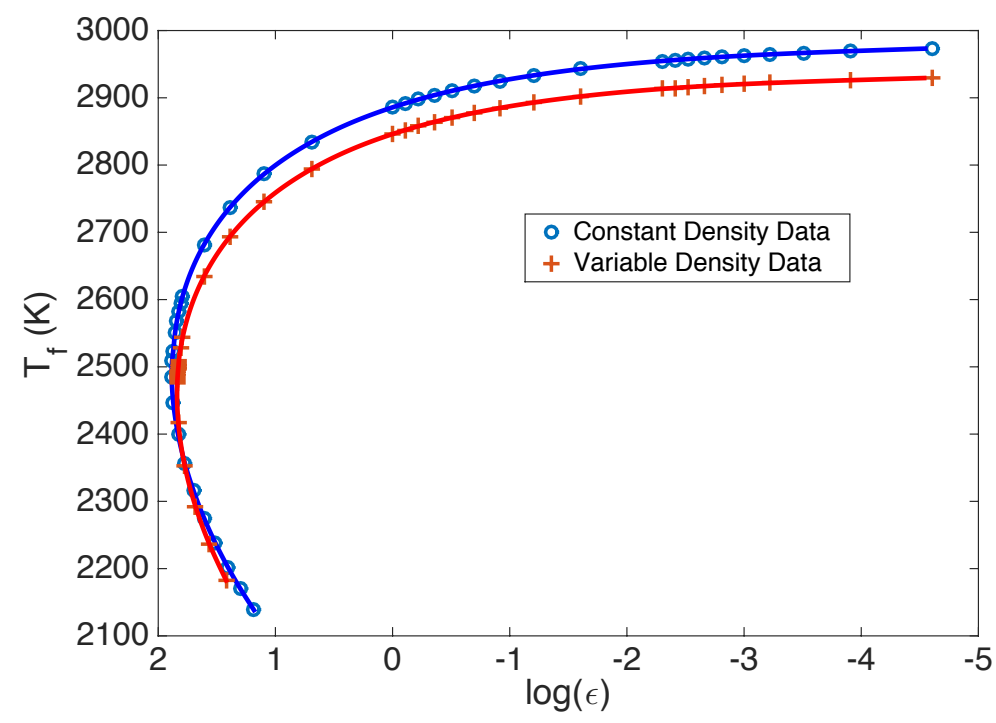

(b) flame temperature

Figure 9. Comparison of flame position and temperature between constant and variable density conditions.

In Fig. 10, we show the degree of reactant leakage through the reaction zone for the constant and variable density formulations. In both cases the mass fractions tend to zero at low strain rates, as it should. Elsewhere, the variable density solution shows a slight deviation in the leakage of $Y_{1}$ and $Y_{2}$ from the constant density solution. This is more 


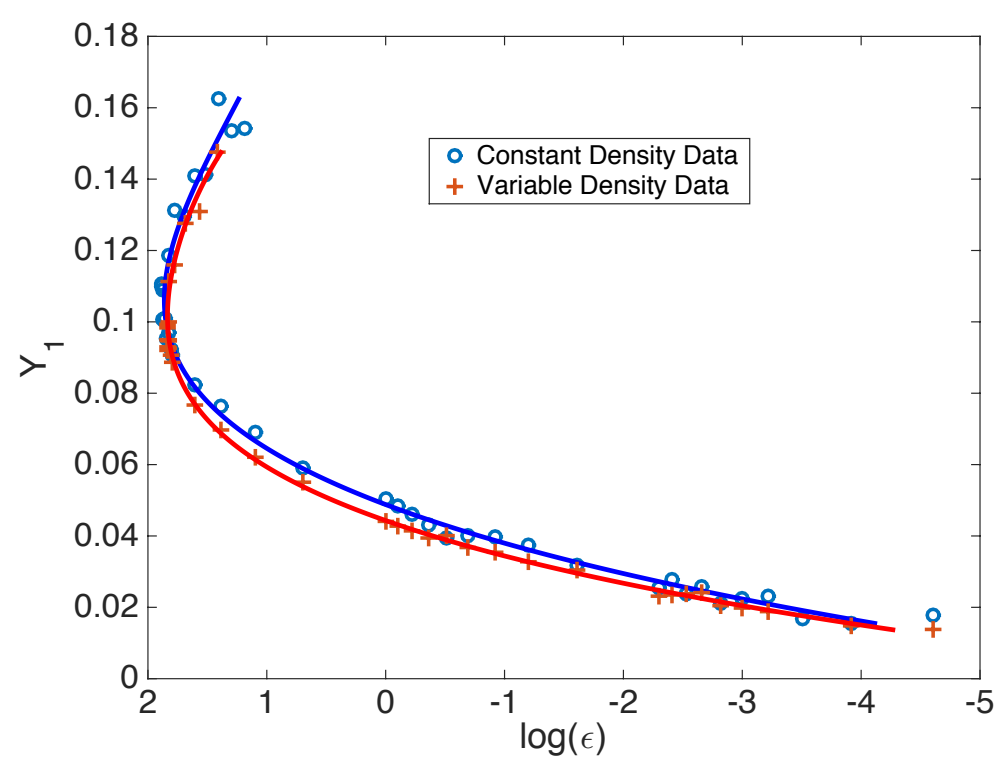

(a) Unconsumed titanium mass fraction

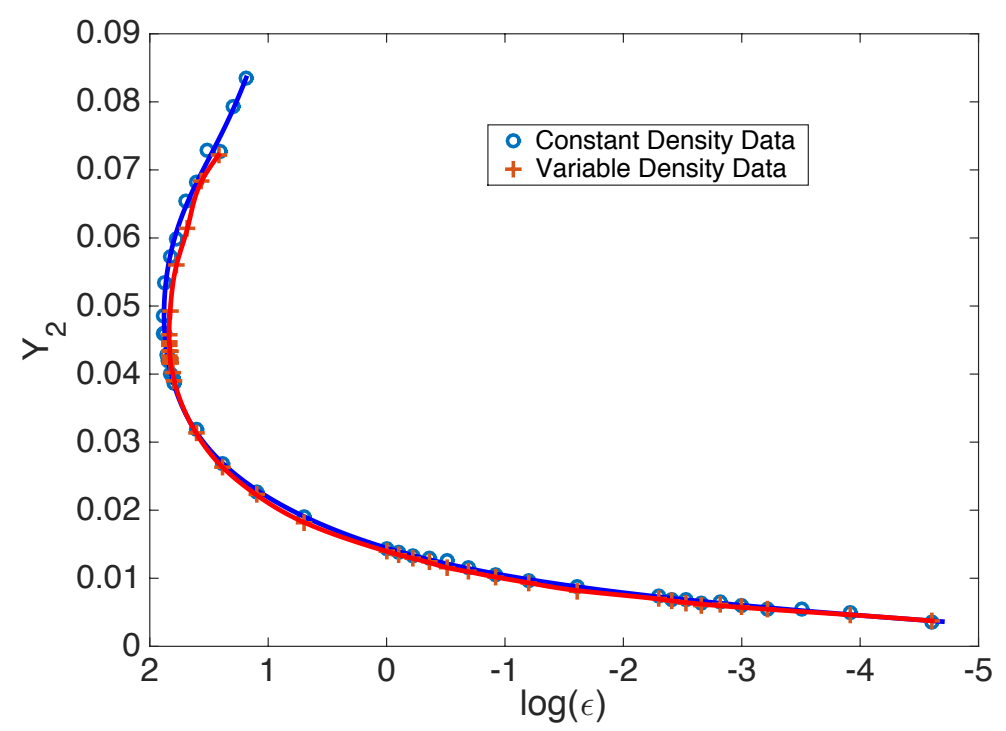

(b) Unconsumed boron mass fraction

Figure 10. $Y_{1}$ response curve comparison between constant and variable density approximations

evident for titanium, because the extent of titanium diffusion is much smaller than that of boron causing a sharper response to density variations. The difference between the two solutions at extinction is nearly 0.005 for $Y_{2}$ and 0.05 for $Y_{1}$, approximately $10 \%$ of the actual extinction values. 


\section{Conclusions}

A multi-component mixture theory is used to describe condensed phase diffusive combustion, in particular for titanium-boron reaction in a counterflow geometry. The Maxwell-Stefan model for multi-component mixtures is informed by the binary diffusivities of any pair of species comprising the mixture. Because of the complications arising from the direct use of the Maxwell-Stefan model in combustion studies, a generalized Fick law is often adopted with coefficients, referred to as Fick diffusivities, which are often assumed to be constant. These coefficients, however, are concentration-dependent and could be related to the binary diffusivities via cumbersome expressions. A significant contribution of this work is the derivation of a multi-component diffusion model for a three-component mixture, which reduces to the gaseous model when molecular weights and diffusivities for each species are equal.

The proposed diffusion expressions can be simplified by assuming equal molecular weights. A comparison between the two diffusion models, with equal and unequal molecular weights, has been carried out in the constant density limit (for simplicity of computations). The response curves constructed numerically show that the two diffusion models lead to identical results at large strain rates as expected, and vary by less than $2 \%$ near extinction. This suggests that one could safely use the constant molecular weight approximation in the proposed diffusion model with minimal loss in accuracy, while saving computational time and complexity.

Low temperature measurements of the boron-titanium diboride binary diffusivity, $\mathscr{D}_{23}$, fall in the range of $10^{-13}$ to $10^{-20} \mathrm{~m}^{2} / \mathrm{s}$ while the titanium-titanium diboride diffusivity, $\mathscr{D}_{13}$, is three orders of magnitude lower. These diffusivities, however, are unavailable at the combustion temperature of Ti-B nano-composites, measured near $3000 \mathrm{~K}$. The derived expressions of flame position and temperature from the fast-chemistry asymptotic analysis have been used to estimate the aforementioned diffusivities in a manner which is consistent with macroscopically observed adiabatic flame temperatures. These estimates may hold in more general circumstances because, as noted from our simulations the flame temperature drops only slightly over the entire range of strain rates sustaining steady combustion.

Combustion characteristics at the interface of two condensed phase reactant streams that form an opposed counterflow are represented by a typical S-shaped response curve. In this paper we only address the portion of the S-curve that extends from a vigorous burning state, where the flame temperature reaches its maximum value (the adiabatic flame temperature) and both titanium and boron are completely consumed, down to an extinction state associated with 
incomplete combustion and with a flame temperature significantly below the adiabatic value. The extinction state is identified when the strain rate reaches a critical value, and for larger strain rates a balance between advection, diffusion and reaction is not possible under steady conditions. The other portion of the S-curve, which extends to exceedingly large values of the strain rate is associated with chemically frozen, or weakly-burning states. Our analysis therefore suggests that in composite materials the distribution of local reaction sites and local frozen sites would depend on the local strain rate distribution.

\section{Acknowledgments}

Supported by the Defense Threat Reduction Agency, HDTRA1-10-1-0020, program manager Dr. S. Peiris. Additional support for MM was provided by Air Force Office of Scientific Research, 917 AFUOFMO C00043936-1, program manager Dr. M. Birkhan, DSS was also supported by ONR, 917 Navy Sub USC DS 2012-03440, Dr. C. Bedford Program manager.

\section{Appendix A.}

The relation between the Fick diffusivities $\mathbb{D}_{i j}$ and binary diffusivities $\mathscr{D}_{i j}$ for a ternary mixture are given by

$$
\begin{aligned}
& \mathbb{D}_{11}=-\frac{\frac{\left(Y_{2}+Y_{3}\right)^{2}}{X_{1} \mathscr{D}_{23}}+\frac{Y_{2}^{2}}{\mathscr{D}_{13} X_{2}}+\frac{Y_{3}^{2}}{X_{3} \mathscr{D}_{12}}}{\frac{X_{1}}{\mathscr{D}_{12} \mathscr{D}_{13}}+\frac{X_{2}}{\mathscr{D}_{12} \mathscr{D}_{23}}+\frac{X_{3}}{\mathscr{D}_{13} \mathscr{D}_{23}}} \\
& \mathbb{D}_{22}=-\frac{\frac{\left(Y_{1}+Y_{3}\right)^{2}}{\mathscr{D}_{13} X_{2}}+\frac{Y_{3}^{2}}{X_{3} \mathscr{D}_{12}}+\frac{Y_{1}^{2}}{X_{1} \mathscr{D}_{23}}}{\frac{X_{1}}{\mathscr{D}_{12} \mathscr{D}_{13}}+\frac{X_{2}}{\mathscr{D}_{12} \mathscr{D}_{23}}+\frac{X_{3}}{\mathscr{D}_{13} \mathscr{D}_{23}}} \\
& \mathbb{D}_{33}=-\frac{\frac{\left(Y_{2}+Y_{1}\right)^{2}}{X_{3} \mathscr{D}_{12}}+\frac{Y_{1}^{2}}{X_{1} \mathscr{D}_{23}}+\frac{Y_{2}^{2}}{X_{2} \mathscr{D}_{13}}}{\frac{X_{1}}{\mathscr{D}_{12} \mathscr{D}_{13}}+\frac{X_{2}}{\mathscr{D}_{12} \mathscr{D}_{23}}+\frac{X_{3}}{\mathscr{D}_{13} \mathscr{D}_{23}}} \\
& \mathbb{D}_{12}=\frac{\frac{Y_{1}\left(Y_{2}+Y_{3}\right)}{X_{1} \mathscr{D}_{23}}+\frac{Y_{2}\left(Y_{1}+Y_{3}\right)}{X_{2} \mathscr{D}_{13}}-\frac{Y_{3}^{2}}{X_{3} \mathscr{D}_{12}}}{\frac{X_{1}}{\mathscr{D}_{12} \mathscr{D}_{13}}+\frac{X_{2}}{\mathscr{D}_{12} \mathscr{D}_{23}}+\frac{X_{3}}{\mathscr{D}_{13} \mathscr{D}_{23}}}
\end{aligned}
$$




$$
\begin{aligned}
& \mathbb{D}_{13}= \frac{\frac{Y_{3}\left(Y_{2}+Y_{1}\right)}{X_{3} \mathscr{D}_{12}}+\frac{Y_{1}\left(Y_{2}+Y_{3}\right)}{X_{1} \mathscr{D}_{23}}-\frac{Y_{2}^{2}}{X_{2} \mathscr{D}_{13}}}{\mathscr{D}_{12} \mathscr{D}_{13}}+\frac{X_{2}}{\mathscr{D}_{12} \mathscr{D}_{23}}+\frac{X_{3}}{\mathscr{D}_{13} \mathscr{D}_{23}} \\
& \mathbb{D}_{23}=\frac{\frac{Y_{2}\left(Y_{1}+Y_{3}\right)}{X_{2} \mathscr{D}_{13}}+\frac{Y_{3}\left(Y_{2}+Y_{1}\right)}{X_{3} \mathscr{D}_{12}}-\frac{Y_{1}^{2}}{X_{1} \mathscr{D}_{23}}}{\frac{X_{1}}{\mathscr{D}_{12} \mathscr{D}_{13}}+\frac{X_{2}}{\mathscr{D}_{12} \mathscr{D}_{23}}+\frac{X_{3}}{\mathscr{D}_{13} \mathscr{D}_{23}}}
\end{aligned}
$$




\section{References}

[1] Advanced energetic materials, committee on Advanced Energetic Materials and Manufacturing Technologies, (2004).

[2] A. S. Rogachev, Exothermic reaction waves in multilayer nanofilms, Russian Chemical Reviews 77 (1) (2015) $21-37$.

[3] T. Weihs, Fabrication and characterization of reactive multilayer films and foils, in Metallic Films for Electronic, Optical and Magnetic Applications, Structure, Processing and Properties, K. Barmak and K. Coffey Eds. (2014) 160-243.

[4] D. Adams, Reactive multilayers fabricated by vapor deposition: A critical review, Thin Solid Film 576 (2015) 98-128.

[5] T. P. Weihs, Self-propogating reactions in multilayer materials, Institute of Physics, 1998, Ch. F7.

[6] G. M. Fritz, H. Joress, T. P. Weihs, Enabling and controlling slow reaction velocities in low-density compacts of multilayer reactive particles, Combustion and Flame 158 (2011) 1084-1088.

[7] I. Sraj, Numerical simulation of shock initiation of ni/al multilayered composites, Journal of Applied Physics 023515 (2014).

[8] N. Glumac, D. S. Stewart, M. D. Clemenson, S. P. Koundinyan, J. B. Bdzil, J. C. Foster, Shock actuated burning of thermitic and inter-metallic reactive composite materialsIn preparation, results presented for Integrated Experimental and Modeling Study of Meso-scale Processes in Reactive Materials, HDTRA-08-10, Defense Threat Reduction Agency Basic Research Technical Review, July $29,2014$.

[9] D. Eakins, N. Thadhani, Mesoscale simulation of the configuration-dependent shock-compression response of $\mathrm{Ni}+\mathrm{Al}$ powder mixture, Acta Materialia 56 (2008) 1496-1510.

[10] D. R. Gaskell, Introduction to the Thermodynamics of Materials, 5th Edition, Taylor and Francis, 2008.

[11] C. F. Curtiss, Symmetric gaseous diffusion coefficients, J. Chem. Phys. 49 (1968) 2917-2919.

[12] C. F. Curtiss, R. B. Bird, Multicomponent diffusion, Ind. Eng. Chem. Res. (1999) 2515-2522.

[13] M. A. Trunov, V. K. Hoffmann, M. Schoenitz, E. L. Dreizin, Combustion of boron-titanium nanocomposite powders in different environments, Journal of Propulsion and Power 24 (2) (2008) 184-191.

[14] J. O. Hirschfelder, C. F. Curtiss, R. B. Bird, Molecular theory of gases and liquids, Wiley, 1954.

[15] R. Taylor, R. Krishna, Multicomponent Mass Transfer, Wiley, 1993.

[16] R. B. Bird, W. E. Stewart, E. N. Lightfoot, Transport Phenomena, Wiley, 2002.

[17] A. Liñán, The asymptotic structure of counterflow diffusion flames for large activation energies, Acta Astronautica 2 (1974) 1009.

[18] S. Cheatham, M. Matalon, A general asymptotic theory of diffusion flames with application to cellular instability, Journal of Fluid Mechanics 414 (2000) 105-144.

[19] Z. Fan, B. Cantor, The kinetics and mechanism of interfacial reaction in sigma fibre-reinforced ti mmcs, Composites Part A 28A (1997) $131-140$

[20] H. Scmidt, Self-diffusion of boron in titanium diboride, J. Appl. Phys 93 (2) (2003) 907-911.

[21] PETSc, version 3.4.3, Argonne National Lab, Lemont, IL, 2013.

[22] V. Kurdyumov, M. Matalon, Dynamics of an edge-flame in a mixing layer, Combustion and Flame 139 (4) (2004) $329-339$.

[23] I. Barin, Thermochemical Data of Pure Substances, Weinheim: VCH, 1995. 
[24] Boron (b) thermal diffusivity and conductivity, beta-rhombohedral boron, in: O. Madelung, U. Rossler, M. Schulz (Eds.), Non-Tetrahedrally Bonded Elements and Binary Compounds I, Vol. 41C of Landolt-Bornstein - Group III Condensed Matter, Springer Berlin Heidelberg, 1998, pp. 1-6.

[25] G. K. White, Thermal conductivity at $273-300 \mathrm{k}$, in: O. Madelung, G. K. White (Eds.), Thermal conductivity of pure metals and Alloys, Vol. 15c, Springer, 2015.

[26] R. Munro, Material properties of titanium diboride, J. Res. Natl. Inst. Stand. Technol 105 (2000) 709-720.

[27] M. Pacheco, Self-sustained High-temperature Reactions: Initiation, propagation and synthesis, Ph.D. thesis, Universidad Carlos III de Madrid (2007).

[28] N. L. Peterson, Diffusion in refractory metals, Tech. Rep. 60-793, Wadd Technical Report (1960).

[29] MATLAB, version 8.4.0 (R2014b), The MathWorks Inc., Natick, Massachusetts, 2014. 